\title{
Abnormal Biodistribution on ${ }^{99 m}$ Tc-Red Blood Cell-Labeled Multigated Acquisition in the Presence of Suspected Cold Agglutinin Disease
}

\author{
Robert Massey and Jennifer S. Jurgens \\ Nuclear Medicine Service, Walter Reed National Military Medical Center, Bethesda, Maryland
}

\begin{abstract}
$A^{99 m}$ Tc-red blood cell (RBC)-labeled multigated acquisition is a procedure in which the patient's RBCs are radiolabeled and imaged with electrocardiography-gated cardiac scintigraphy to assess the heart's pumping efficiency. Cold agglutinin disease, or cold antibody autoimmune hemolytic anemia, is a rare form of autoimmune hemolytic anemia in which the body's immune system attacks and destroys its own RBCs. This case addresses an altered biodistribution pattern of radiolabeled RBCs in the presence of suspected cold agglutinin disease observed during a multigated acquisition.
\end{abstract}

Key Words: cardiology; hematology; radiopharmaceuticals; cold

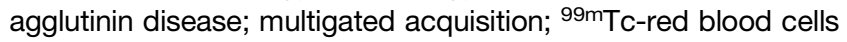

J Nucl Med Technol 2019; 47:175-176

DOI: 10.2967/jnmt.118.222182

\section{$\mathbf{I}$} is not uncommon for lymphoma patients to present with autoimmune hemolytic anemia. Although this condition is most commonly associated with warm autoantibodies, it has also been reported less frequently from cold agglutinin disease and there are even fewer reports of diffuse large Bcell lymphoma presenting as cold agglutinin disease. In cold agglutinin disease, antibodies bind to the red blood cell (RBC) membrane, resulting in erythrocyte hemolysis. Naturally, these damaged RBCs will be preferentially sequestered by the spleen. As a result, elevated cold agglutinin titers may cause an altered ${ }^{99 \mathrm{~m}} \mathrm{Tc}-\mathrm{RBC}$ biodistribution pattern resulting in a nondiagnostic multigated acquisition study. This is a rarely reported event and not well known in the molecular imaging community. The imaging findings of this suspected unusual occurrence are reviewed in this presentation.

\section{CASE REPORT}

A 53-y-old man with a history of diffuse large B-cell lymphoma, 4 y after autologous stem cell transplantation, was

Received Oct. 25, 2018; revision accepted Dec. 14, 2018.

For correspondence or reprints contact: Robert Massey, Walter Reed National Military Medical Center, 8901 Rockville Pike, Bethesda, MD 20889.

E-mail: robert.massey.ctr@mail.mil

Published online Jan. 29, 2019.

COPYRIGHT (c) 2019 by the Society of Nuclear Medicine and Molecular Imaging. referred for an annual ${ }^{99 \mathrm{~m}} \mathrm{Tc}$-multigated acquisition evaluation. The patient had 6 other previous multigated acquisition studies dating from July 2012 to December 2015 with ejection fractions ranging from a high of $53 \%$ to a low of $39 \%$. Previous studies revealed no unusual or abnormal ${ }^{99 \mathrm{~m} T c-R B C}$ radiopharmaceutical biodistribution or altered physiologic uptake. However, the patient's most recent multigated acquisition was deemed uninterpretable because of poor cardiac blood-pool localization. The test was repeated the following day with the same uninterpretable results. Although there was a negligible amount of blood-pool activity, there was an overwhelming amount of activity concentrated within the liver and spleen (Fig. 1), consistent with reticuloendothelial system

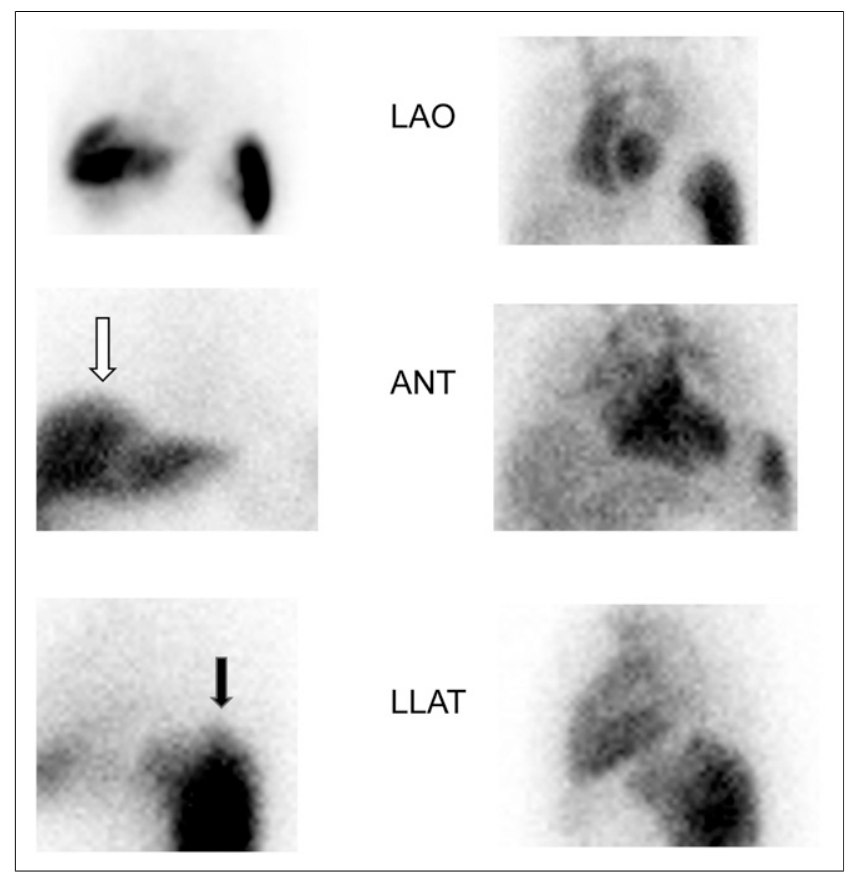

FIGURE 1. Multigated acquisition images demonstrating altered biodistribution (left) and normal biodistribution of standard blood pool (right) from 3 planes (from top to bottom: left anterior oblique, anterior, and left lateral). These images were acquired from same patient at 2 different time points. Altered biodistribution demonstrates prominent activity within liver (open arrow) and spleen (solid arrow), with lack of standard blood-pool activity demonstrated normally. 
uptake of colloid. Medication reconciliation demonstrated no identifiable causative interaction, and no previous nuclear medicine studies had been performed. The in vitro UltraTag (Mallinckrodt) RBC labeling kit was prepared per the manufacturer's guidelines, and the quality of the radiopharmaceutical was acceptable (99\%). Current laboratory studies were remarkable for a slightly elevated anion gap (19 mmol/L) and total bilirubin $(1.4 \mathrm{mg} / \mathrm{dL})$, a slightly decreased platelet count $(155,000 / \mu \mathrm{L})$, and a highly elevated herpes simplex virus type 1 IgG type-specific (26.70). A thorough literature search revealed no explanation for this anomaly, with the exception of a SlideShare presentation that showed a similar multigated acquisition ${ }^{99 \mathrm{~m}} \mathrm{Tc}-\mathrm{RBC}$ distribution pattern in a patient who had cold agglutinin disease ( 1 ). The most recent screening and detection of cold agglutinin disease that was noted in the available lab reports was on June 13, 2012.

\section{DISCUSSION}

Cold agglutinin disease is a rare autoimmune disorder in which autoantibodies produced by a person's immune system mistakenly target and destroy RBCs, causing hemolytic anemia.

Primary cold agglutinin disease is a well-recognized complication of lymphoproliferative disorders, with lymphoplasmacytic lymphoma being the most common subtype of malignant lymphoma whereas diffuse large B-cell lymphoma is less well associated. Cold agglutinin disease may also be secondary to infections such as HIV. Although there are some well-known explanations for alterations in ${ }^{99 \mathrm{~m}} \mathrm{Tc}$ $\mathrm{RBC}$ radiopharmaceutical biodistribution (2), no reports in the literature directly link an abnormal ${ }^{99} \mathrm{~m} \mathrm{Tc}-\mathrm{RBC}$ distribution to cold agglutinin disease. However, in view of a similar incident reported in the literature (1), we suspected cold agglutinin disease as the likely culprit in this nondiagnostic multigated acquisition.

\section{CONCLUSION}

Multigated acquisition cardiac assessments are commonly performed for anthracycline-based chemotherapy patients with a history of diffuse large B-cell lymphoma. Although there are well-recognized explanations for some cases of altered ${ }^{99 \mathrm{~m}}$ Tc-RBC radiopharmaceutical biodistribution, some other cases cannot be so easily explained. The presence of cold agglutinin disease should be investigated in these patients whenever an unexpected biodistribution pattern is observed.

\section{DISCLOSURE}

The views expressed in this article are those of the authors and do not reflect the official policy of the Department of the Army, Navy, or Air Force; the Department of Defense; or the U.S. Government. No potential conflict of interest relevant to this article was reported.

\section{REFERENCES}

1. Barron BJ, Joseph UA, Wan DQ, Rasberry DL. Mysterious radiopharmaceutical localization: a pictorial interactive exhibit. https://www.slideshare.net/brucelee55/ mysterious-radiopharmaceutical-localization-a. Published October 27, 2010. Accessed February 21, 2019

2. Hambye AS, Vandermeiren R, Vervaet A, Vandevivere J. Failure to label red blood cells adequately in daily practice using an in vivo method: methodological and clinical considerations. Eur J Nucl Med. 1995;22:61-67. 